\section{TATRA \\ MOUNTaiNS \\ Mathematical Publications}

DOI: $10.2478 /$ tmmp-2019-0002

Tatra Mt. Math. Publ. 73 (2019), 9-18

\title{
ON PARTITIONS AND PERIODIC SEQUENCES
}

\author{
MiLAN PAŠTÉKA
}

Department of Mathematics, Faculty of Education, University of Trnava, Trnava, SLOVAKIA

\begin{abstract}
In the first part we associate a periodic sequence with a partition and study the connection the distribution of elements of uniform limit of the sequences. Then some facts of statistical independence of these limits are proved.
\end{abstract}

\section{Introduction}

This paper is inspired by the papers [CIV], CV], $\mathrm{K}$ ] where the uniformly distributed sequences of partitions of the unit interval are studied. For technical reasons we will study the systems of finite sequences of the unit interval — an equivalent of partitions.

Let $V_{N}=\left\{v_{N}(1), \ldots, v_{N}\left(B_{N}\right)\right\}, N=1,2,3, \ldots, \lim _{N \rightarrow \infty} B_{N}=\infty$ be a system of finite sequences of elements of interval $[0,1]$. We say that this system is uniformly distributed if and only if

$$
\lim _{N \rightarrow \infty} \frac{1}{B_{N}} \sum_{n=1}^{B_{N}} f\left(v_{N}(n)\right)=\int_{0}^{1} f(x) \mathrm{d} x .
$$

By the standard way we can prove

TheOREM 1. The system $V_{N}, N=1,2,3, \ldots$ is uniformly distributed if and only if

$$
\lim _{N \rightarrow \infty} \frac{1}{B_{N}}\left|\left\{n \leq B_{N} ; v_{N}(n)<x\right\}\right|=x
$$

for every $x \in[0,1]$.

(C) 2019 Mathematical Institute, Slovak Academy of Sciences. 2010 Mathematics Subject Classification: 11A25.

Keywords: statistical independence, uniform distribution, polyadic continuity.

Supported by Vega Grant 2/0109/18.

Licensed under the Creative Commons Attribution-NC-ND4.0 International Public License. 


\section{MILAN PAŠTÉKA}

The notion of the uniformly distributed sequence was first introduced by H. W e y l in his famous paper [WEY]. An equivalent of this definition is that a sequence $v$ of elements of interval $[0,1]$ is uniformly distributed if and only if the system of finite sequences $\{v(1), \ldots, v(N)\}, N=1,2,3, \ldots$ is uniformly distributed. This means that the sequence $\{v(n)\}$ is uniformly distributed if and only if the equality

$$
\lim _{N \rightarrow \infty} \frac{1}{N}|\{n \leq N ; v(n)<x\}|=x
$$

holds for arbitrary $x \in[0,1]$. This can be formulated in an equivalent form: Let $\mathbb{N}$ be the set of positive integers. Denote by $\mathcal{D}$ the system of sets $A \subset \mathbb{N}$ such that the limit $d(A):=\lim _{N \rightarrow \infty} \frac{1}{N}|\{n \leq N ; n \in A\}|$ exists. In this case we say that the set $A$ has asymptotic density and we call the value $d(A)$ the asymptotic density of $A$. For details, we refer the reader to $[\mathrm{PAS}$. Clearly, the sequence $\{v(n)\}$ is uniformly distributed if and only if $\{n \in \mathbb{N} ; v(n)<x\} \in \mathcal{D}$ and $d(\{n \in \mathbb{N} ; v(n)<x\})=x$ for each $x \in[0,1]$.

In 1946 R. C. B u c k [BUC] constructed the measure density. He started from the asymptotic density and applied some methods of measure theory. Denote $r+(m)=\{n \in \mathbb{N} ; n \equiv r(\bmod m)\}$ - the arithmetic progression, where $m \in \mathbb{N}$, $r=0,1,2, \ldots, 0+(m):=(m)$. If $A \subset \mathbb{N}$, then we shall call the value

$$
\mu^{*}(A)=\inf \left\{\sum_{j=1}^{k} \frac{1}{m_{j}} ; A \subset \bigcup_{j=1}^{k} r_{j}+\left(m_{j}\right)\right\}
$$

the Buck's measure density of $A$. A set $A \subset \mathbb{N}$ is called Buck measurable if and only if $\mu^{*}(A)+\mu^{*}(\mathbb{N} \backslash A)=1$. Denote $\mathcal{D}_{\mu}$ the system of all Buck measurable sets. Then $\mathcal{D}_{\mu}$ is an algebra of sets and $\mu=\left.\mu^{*}\right|_{\mathcal{D}_{\mu}}$ is a finitely additive probability measure on $\mathcal{D}_{\mu}$. For the details, we refer the reader to [PAS]. A sequence of elements of $[0,1]$ is called Buck uniformly distributed if and only if $\{n \in \mathbb{N} ; v(n)<x\} \in \mathcal{D}_{\mu}$ and $\mu(\{n \in \mathbb{N} ; v(n)<x\})=x$ for all $x \in[0,1]$. (See also [PAS2].)

We say that a sequence $v$ is polyadicly continuous on the set $A \subset \mathbb{N}$ if and only if for each $\varepsilon>0$ there exists a positive integer $m$ so that for all $n_{1}, n_{2} \in A$ we have

$$
n_{1} \equiv n_{2} \quad(\bmod m) \Longrightarrow\left|v\left(n_{1}\right)-v\left(n_{2}\right)\right|<\varepsilon .
$$

We say that $v$ is polyadicly continuous if and only if it is polyadicly continuous on $\mathbb{N}$.

Denote for a sequence $v$ and $N$-positive integer

$$
E_{N}(v)=\frac{1}{N} \sum_{n=1}^{N} v(n) .
$$




\section{ON PARTITIONS AND PERIODIC SEQUENCES}

If there exists the proper limit

$$
\lim _{N \rightarrow \infty} E_{N}(v):=E(v),
$$

then we say that $v$ has a mean value and $E(v)$ is called the mean value of $v$. (In literature this is also known as $(C, 1)$ summable sequence and $(C, 1)$ limit.)

We say that a sequence of positive integers $\left\{k_{n}\right\}$ is uniformly distributed in $\mathbb{Z}$ if and only if for every $m \in \mathbb{N}$ and $r=0,1, \ldots$ the set $\left\{n \in \mathbb{N} ; k_{n} \in r+(m)\right\}$ belongs to $\mathcal{D}$ and its asymptotic density is $\frac{1}{m}$. (First introduced and studied by I. Niven in the paper $[\mathrm{N}]$ ).

Theorem 2. A sequence $v$ of elements $[0,1]$ is Buck uniformly distributed if and only if for each sequence $\left\{k_{n}\right\}$ of positive integers uniformly distributed in $\mathbb{Z}$ the equality

$$
\lim _{N \rightarrow \infty} E_{N}\left(g\left(v\left(k_{n}\right)\right)\right)=\int_{0}^{1} g(x) \mathrm{d} x
$$

holds for every continuous function $g$ defined on $[0,1]$.

For the proof we refer the reader to [PAS, p. 122].

\section{Almost polyadic continuity and almost uniform convergence}

The following two propositions proved in [PAS, pp. 106-108] will be useful for the next results.

Proposition 1. Let $v$ be a periodic sequence with the period $B \in \mathbb{N}$. Then $v$ has the mean value

$$
E(v)=\sum_{j=1}^{B} v(j)
$$

and for each sequence $\left\{k_{n}\right\}$ uniformly distributed in $\mathbb{Z}$ we have

$$
\lim _{N \rightarrow \infty} \frac{1}{N} \sum_{n=1}^{N} v\left(k_{n}\right)=E(v) .
$$

Every polyadicly continuous sequence can be uniformly approximated by the periodic functions thus we have

Proposition 2. Each polyadicly continuous sequence $v$ has a mean value and

$$
\lim _{N \rightarrow \infty} \frac{1}{N} \sum_{n=1}^{N} v\left(k_{n}\right)=E(v)
$$

for each sequence $\left\{k_{n}\right\}$ of positive integers $\left\{k_{n}\right\}$ uniformly distributed in $\mathbb{Z}$. 
A sequence $v$ will be called almost polyadicly continuous if and only if for every $\delta>0$ there exists a Buck measurable set $B \subset \mathbb{N}$ that $\mu(B) \leq \delta$ and $v$ is polyadicly continuous on the set $\mathbb{N} \backslash B$.

Theorem 3. If $v$ is a bounded almost polyadicly continuous sequence, then $v$ has a mean value and for each sequence of positive integers $\left\{k_{n}\right\}$ uniformly distributed in $\mathbb{Z}$ we have

$$
\lim _{N \rightarrow \infty} \frac{1}{N} \sum_{n=1}^{N} v\left(k_{n}\right)=E(v) .
$$

Pro of. Suppose that $v$ is an almost polyadicly continuous sequence and $C>0$ is such contant that $|v(n)|<C, n \in \mathbb{N}$. Consider $\delta>0$. Then there exists such $m \in N, r_{1}, \ldots, r_{k} \in\{0, \ldots, m-1\}$ that $\frac{k}{m}>1-\varepsilon$ and $v$ is polyadicly continuous on the set $A=\cup_{j=1}^{k} r_{j}+(m)$. Define a sequence $\left\{v_{0}(n)\right\}$ in the following way: $v_{0}(n)=v(n)$ for $n \in A$ and $v_{0}(n)=0$ otherwise. Clearly,

$$
\left|E_{N}(v)-E_{N}\left(v_{0}\right)\right| \leq \frac{C}{N} \sum_{\substack{n \leq N, n \notin A}} 1, \quad N=1,2,3, \ldots
$$

We see immediately that the sequence $\left\{v_{0}(n)\right\}$ is polyadicly continuous thus it has a mean value. Thus, from the inequality above, we get that the distance between the upper and lower limit of $E_{N}(v)$ is smaller than $2 C \delta$. For $\delta \rightarrow 0^{+}$ we get that the $\lim _{N \rightarrow \infty} E_{N}(v)$ exists. The same reasons provide also the equality (1).

By combining Theorem 3 and Theorem 2 we get:

TheOrem 4. An almost polyadicly continuous sequence of elements of $[0,1]$ is Buck uniformly distributed if and only if it is uniformly distributed.

We say that a system of sequences $v_{N}$ converges almost uniformly for $N \rightarrow \infty$ to sequence $v$ if and only if for every $\delta>0$ there exists a Buck measurable set $S \subset \mathbb{N}$, where $\mu(S)<0$ and $v_{N}$ converges uniformly for $N \rightarrow \infty$ to $v$ on the set $\mathbb{N} \backslash S$.

Proposition 3. If $v_{N}, N=1,2,3, \ldots$ is a system of polyadicly continuous sequences of elements of $[0,1]$ and it converges almost uniformly to a sequence $v$, then $v$ is almost polyadicly continuous and

$$
\lim _{N \rightarrow \infty} E\left(v_{N}\right)=E(v) .
$$

Each finite sequence $V_{N}, N=1,2,3, \ldots$ can be extended to a periodic sequence $\omega_{N}$ in the following way

$$
\omega_{N}(n)=v_{N}(j) \Longleftrightarrow n \equiv j\left(\bmod B_{N}\right), \quad j=1, \ldots, B_{N}, \quad n \in \mathbb{N} .
$$




\section{ON PARTITIONS AND PERIODIC SEQUENCES}

From Theorem 4 and Proposition 3 we get immediately

Theorem 5. Let the system of sequences $\omega_{N}$ converge almost uniformly to a sequence $\omega$ for $N \rightarrow \infty$ uniformly for $n \in \mathbb{N}$. Then the system of finite sequences $V_{N}, N=1,2,3, \ldots$ is uniformly distributed if and only if the sequence $\omega$ is Buck uniformly distributed.

The following fact can be proved directly from Cauchy Bolzano criterion of uniform convergence:

Proposition 4. Let $\sum_{N=1}^{\infty} a_{N}$ be a convergent series with positive elements. Suppose that $\left\{\alpha_{N}(n)\right\}, N=1,2,3, \ldots$ is system of sequences that

$$
\left|\alpha_{N}(n)-\alpha_{N+1}(n)\right| \leq a_{N}, \quad n \in \mathbb{N} .
$$

Then $\left\{\alpha_{N}(n)\right\}$ converges uniformly to a suitable sequence $\{\alpha(n)\}$.

EXAMPLE 1. Let $\left\{B_{N}\right\}$ be an increasing sequence of positive integers such that $B_{N} \mid B_{N+1}, N=1,2,3, \ldots$ Then the series $\sum_{N=1}^{\infty} \frac{1}{B_{N}}$ converges. If we construct a system of periodic sequences $\left\{\omega_{N}(n)\right\}$, where $\omega_{N}(n)$ is periodic modulo $B_{N}, N=1,2,3, \ldots$ such that

$$
r \equiv n\left(\bmod B_{N}\right) \Longrightarrow\left|\omega_{N}(r)-\omega_{N+1}(n)\right| \leq \frac{c}{B_{N}}
$$

for $n, r \in \mathbb{N}$ and $N=1,2,3, \ldots$ for some $c>0$, then $\left\{\omega_{N}(n)\right\}$ converges to a suitable polyadicly continuous sequence for $N \rightarrow \infty$ uniformly for $n \in \mathbb{N}$.

\section{Statistical independence}

This notion is introduced in $\mathrm{Ra}$. Let $v_{1}, \ldots, v_{n}$ be sequences of elements of $[0,1]$. We say that these sequences are statistically independent if and only if for continuous functions $g_{1}, \ldots, g_{k}$ defined on $[0,1]$ and the sequence $u=g\left(v_{1}\right) \ldots$ $\ldots g_{k}\left(v_{k}\right)$ we have

$$
E_{N}(u)-E_{N}\left(g\left(v_{1}\right)\right) \ldots E_{N}\left(g_{k}\left(v_{k}\right)\right) \rightarrow 0
$$

for $N \rightarrow \infty$.

For almost polyadicly continuous sequences this has the following more simple form

Proposition 5. If $v_{1}, \ldots, v_{k}$ are almost polyadicly continuous sequences of elements of $[0,1]$ and $u$ has the same meaning as above, then they are statistically independent if and only if

$$
E(u)=E\left(g_{1}\left(v_{1}\right)\right) \ldots E\left(g_{k}\left(v_{k}\right)\right)
$$

for each function $g_{1}, \ldots, g_{k}$ continuous on $[0,1]$. 


\section{MILAN PAŠTÉKA}

Proposition 6. Let $\left\{v_{1}(n)\right\}$ be a periodic sequence with the period $M_{1}$ and $\left\{v_{2}(n)\right\}$ be a periodic sequence with the period $M_{2}$. If $\left(M_{1}, M_{2}\right)=1$, then

$$
E\left(v_{1} v_{2}\right)=E\left(v_{1}\right) E\left(v_{2}\right) .
$$

P r o of. From the Chinese reminder theorem we get that for each

$$
r_{1} \in\left\{1, \ldots, M_{1}\right\} \quad \text { and } r_{2} \in\left\{1, \ldots, M_{2}\right\}
$$

there exists uniquely determined $r \in\left\{1, \ldots, M_{1} M_{2}\right\}$ such that $r \equiv r_{1}\left(\bmod M_{1}\right)$, $r \equiv r_{2}\left(\bmod M_{1}\right)$. Thus

$$
E\left(v_{1} v_{2}\right)=\frac{1}{M_{1} M_{2}} \sum_{r_{1}, r_{2}} v_{1}\left(r_{1}\right) v_{2}\left(r_{2}\right)=E\left(v_{1}\right) E\left(v_{2}\right) .
$$

If $v$ is a periodic sequence, then for each function $g$ defined on the set of values of $v$ the sequence $g(v)$ is also periodic. And so from Proposition 6 we can conclude:

Proposition 7. If $v_{1}, \ldots, v_{n}$ are periodic sequences with mutually relative prime periods, then these sequences are statistically independent.

Every continuous function on $[0,1]$ is uniformly continuous. This implies that if the system of sequences $v_{N}$, with elements from $[0,1]$, converges almost uniformly to a sequence $v$ for $N \rightarrow \infty$ and $g$ is a continuous function on $[0,1]$, then $g\left(v_{N}\right)$ converges uniformly to $g(v)$ also. Therefore Proposition 3 implies:

Proposition 8. If $v_{N}^{(j)}, N=1,2,3, \ldots$ is such a system of polyadicly continuous sequences, $j=1, \ldots, k$ that for each $N=1,2,3, \ldots$ the sequences $v_{N}^{(j)}, j=1, \ldots, k$ are statistically independent and for each $j=1, \ldots, k$ the system of sequences $v_{N}^{(j)}, N=1,2,3, \ldots$ converges almost uniformly to a sequence $v^{(j)}$, then the sequences $v^{(j)}, j=1, \ldots, k$ are statistically independent.

Suppose that $P$ is some set of primes and $S(P)$ is the semigroup generated by $P$. We say that a sequence $v$ is polyadicly continuous with respect to $P$ if and only if for each $\varepsilon>0$ there exists $m \in S(P)$ so that

$$
n_{1} \equiv n_{2} \quad(\bmod m) \Longrightarrow\left|v\left(n_{1}\right)-v\left(n_{2}\right)\right|<\varepsilon
$$

for $n_{1}, n_{2} \in \mathbb{N}$. It is easy to see that in this case the sequence $v$ is a uniform limit of periodic sequences with periods belonging to $S(P)$. Thus we obtain

Theorem 6. Let $P_{1}, \ldots, P_{k}$ be disjoint sets of primes. If $v_{1}, \ldots, v_{k}$ are such sequences that $v_{j}$ is polyadicly continuous with respect to $P_{j}, j=1, \ldots, k$, then the sequences $v_{1}, \ldots, v_{k}$ are statistically independent. 


\section{ON PARTITIONS AND PERIODIC SEQUENCES}

EXAmple 2. If $P_{1}, \ldots, P_{k}$ are disjoint sets of primes and $s_{j}>1$, then we can define for $n \in \mathbb{N}$

$$
\alpha_{j}(n)=\prod_{\substack{p \mid n, p \in P_{j}}}\left(1-\frac{1}{p^{s_{j}}}\right), \quad j=1, \ldots, k .
$$

The condition $s_{j}>1$ provides that $\alpha_{j}$ is polyadicly continuous with respect to $P_{j}$ for $j=1, \ldots, k$. Thus these sequences are statistically independent.

EXAMPLE 3 . If $m_{1}, \ldots, m_{k}$ are mutually relatively prime positive integers and we define the sequence $v_{j}(n)$ as a Van der Corput sequence with the base $m_{j}$, i.e. for $n=a_{r} m_{j}^{r}+\cdots+a_{1} m_{j}+a_{0}, 0 \leq a_{i} \leq m_{j}-1$ we set

$$
v_{j}(n)=\frac{a_{r}}{m_{j}^{r+1}}+\cdots+\frac{a_{1}}{m_{j}^{2}}+\frac{a_{0}}{m_{j}},
$$

then the sequences $v_{1}, \ldots, v_{k}$ are statistically independent.

\section{Construction of uniformly distributed partitions}

Suppose now (without loss of generality) that the finite sequences $V_{N}, N=$ $1,2,3 \ldots$ are increasing and

$$
\lim _{N \rightarrow \infty} v_{N}(1)=0
$$

Let $1=j_{1}^{N}, \ldots, j_{k_{N}}^{N}$ be an increasing subsequence of $\left\{1, \ldots, B_{N}\right\}$. Then the sequence $V_{N}$ can be decomposed into disjoint subsequences

$$
V_{N}=V(1, N) \cup \cdots \cup V\left(j_{k_{N}}^{N}, N\right),
$$

where $V(k, N)$ is a sequence of consecutive elements of $V_{N}$ with the smallest element $v_{N}\left(j_{k}\right), k=1, \ldots, k_{N}$. For $1 \leq k<k_{N}$ let us denote

$$
\ell(k, N)=v_{N}\left(j_{k+1}^{N}\right)-v_{N}\left(j_{k}^{N}\right),
$$

and for $k=k_{N}$ denote

$$
\ell\left(k_{N}, N\right)=1-v_{N}\left(j_{k_{N}}^{N}\right) .
$$

Then (2) implies

$$
\lim _{N \rightarrow \infty} \sum_{k=1}^{k_{N}} \ell(k, N)=1 .
$$

Theorem 7. Assume that

and

$$
\lim _{N \rightarrow \infty} \ell(k, N)=0
$$

$$
\lim _{N \rightarrow \infty} \frac{|V(k, N)|}{\ell(k, N) B_{N}}=1
$$




\section{MILAN PAŠTÉKA}

converge uniformly for $k$. Then the system of finite sequences $V_{N}, N=1,2,3, \ldots$ is uniformly distributed.

P r o of. Let $x \in(0,1)$ be a fixed number. Then conditions (2) and (4) provide that there exists a positive integer $s_{N}$ so that

$$
\sum_{j=1}^{s_{N}} \ell(j, N) \leq x<\sum_{j=1}^{s_{N}+1} \ell(j, N)
$$

with the exception of a finite number of $N$. This yields

$$
\lim _{N \rightarrow \infty} \sum_{j=1}^{s_{N}} \ell(j, N)=x .
$$

Clearly,

$$
\sum_{j=1}^{s_{N}}|V(j, N)| \leq\left|\left\{n \leq B_{N} ; v_{N}(j) \leq x\right\}\right|<\sum_{j=1}^{s_{N}+1}|V(j, N)| .
$$

From the condition (5) we get that for $\varepsilon>0$ there exists $N_{0}$ such that for $N \geq N_{0}$ and $1 \leq k \leq B_{N}$ we have

$$
(1-\varepsilon) \ell(k, N) B_{N} \leq|V(k, N)| \leq(1-\varepsilon) \ell(k, N) B_{N}
$$

and by substitution in (7) we obtain

$$
(1-\varepsilon) \sum_{j=1}^{s_{N}} \ell(j, N) \leq \frac{\left|\left\{n \leq B_{N} ; v_{N}(j) \leq x\right\}\right|}{B_{N}} \leq(1+\varepsilon) \sum_{j=1}^{s_{N}+1} \ell(j, N) .
$$

From this and (6) we can conclude

$$
\lim _{N \rightarrow \infty} \frac{\left|\left\{n \leq B_{N} ; v_{N}(j) \leq x\right\}\right|}{B_{N}}=x .
$$

Denote

$$
M_{N}=\max \left\{|V(k, N)| ; k=1, \ldots, k_{N}\right\}, m_{N}=\min \left\{|V(k, N)| ; k=1, \ldots, k_{N}\right\}
$$

and

$$
L_{N}=\max \left\{\ell(k, N) ; k=1, \ldots, k_{N}\right\}, \quad \ell_{N}=\min \left\{\ell(k, N) ; k=1, \ldots, k_{N}\right\} .
$$

TheOrem 8. If

$$
\lim _{N \rightarrow \infty} L_{N}=0
$$

and

$$
\lim _{N \rightarrow \infty} \frac{M_{N} \ell_{N}}{m_{N} L_{N}}=1
$$

then the system of finite sequences $V_{N}, N=1,2,3, \ldots$ is uniformly distributed. 


\section{ON PARTITIONS AND PERIODIC SEQUENCES}

Pr o of. We apply Theorem [7. Condition (8) implies that condition (4) is fulfilled.

Clearly, the inequalities

$$
\frac{m_{N}}{L_{N} B_{N}} \leq \frac{|V(k, N)|}{\ell(k, N) B_{N}} \leq \frac{M_{N}}{\ell_{N} B_{N}}, \quad k=1, \ldots, k_{N_{j}}
$$

hold. Thus as a proof that condition (5) holds, it suffices to prove that

$$
\lim _{N \rightarrow \infty} \frac{M_{N}}{\ell_{N} B_{N}}=1
$$

because condition (9) provides that the term on the left hand side of inequalities (10) has the same limit points as the right hand side term. Suppose that the right hand side term has a limit point smaller than 1 . Then for suitable $\alpha<1$ and infinite sequence $\left\{N_{j}\right\}$ we have

$$
\left|V\left(k, N_{j}\right)\right| \leq \alpha \ell\left(k, N_{j}\right) B_{N_{j}}, \quad k=1, \ldots, k_{N_{j}}
$$

and so we get the contradiction

$$
\begin{aligned}
B_{N_{j}} & =\sum_{k=1}^{k_{N_{j}}}\left|V\left(k, N_{j}\right)\right| \\
& \leq \alpha \sum_{k=1}^{k_{N_{j}}} \ell\left(k, N_{j}\right) B_{N_{j}} \leq \alpha B_{N_{j}} .
\end{aligned}
$$

If the right hand side term has a limit point greater than 1 , then the left hand side term has the same limit point and so for suitable $\beta>1$ and an infinite sequence $\left\{N_{j}\right\}$ the inequalities

$$
\left|V\left(k, N_{j}\right)\right| \geq \beta \ell\left(k, N_{j}\right) B_{N_{j}}, \quad k=1, \ldots, k_{N_{j}}
$$

hold. This yields

$$
B_{N_{j}}=\sum_{k=1}^{k_{N_{j}}}\left|V\left(k, N_{j}\right)\right| \geq \beta \sum_{k=1}^{k_{N_{j}}} \ell\left(k, N_{j}\right) B_{N_{j}}
$$

and so

$$
1 \geq \beta \sum_{k=1}^{k_{N_{j}}} \ell\left(k, N_{j}\right)
$$

Therefore,

$$
\lim _{j \rightarrow \infty} \sum_{k=1}^{k_{N_{j}}} \ell\left(k, N_{j}\right) \leq \frac{1}{\beta}<1 \quad \text { - a contradiction with (3) } .
$$




\section{MILAN PAŠTÉKA}

\section{REFERENCES}

[BUC] BUCK, R. C.: The measure theoretic approach to density, Amer. J. Math. 68 (1946), 560-580.

[CIV] CARBONE, I.-IACO, M. R.-VOLCIC, A.: A dynamical system approach to the Kakutani-Fibonacci sequence, Ergodic Theory Dynam. Systems 34 (2014), 1794-1806.

[CV] CARBONE, I.-VOLCIC, A.: A von Neumann theorem for uniformly distributed sequences of partitions, Rend. Circ. Mat. Palermo (2) 60 (2011), 83-88.

[D-T] DRMOTA, M.-TICHY, R. F.: Sequences, Discrepancies and Applications. In: Lecture Notes in Math., Vol. 1651, Springer, Berlin, 1997.

[DTS] DRMOTA, M.-TICHY, R. F.-STRAUCH, O.: LP-discrepancy and statistical independence of sequences, Czechoslovak Math. J. 49 (1999), 97-110.

$[\mathrm{K}] \quad$ KAKUTANI, S.: A problem of equidistribution on the unit interval $[0,1]$, in: Measure Theory, Proc. Conf. Oberwolfach 1975 (A. Bellow and D. Kölzow, eds.), Lecture Notes in Math., Vol. 541, Springer, Berlin, 1976, pp. 369-375.

[N] NIVEN, I.: Uniform distribution of sequences of integers, Trans. Amer. Math. Soc. 98 (1961), 52-61.

[P] POSTNIKOV, R. G.: Introduction to Analytic Number Theory. Nauka, Moscow, 1971; English transl.: Amer. Math. Soc., Vol. 68, Providence, RI, 1981. (In Russian)

[PAS] PAŠTÉKA, M.: Density and related topics, Veda, Bratislava; Academia, Prague, 2017.

[PAS1] _ On four approaches to density, Veda, Bratislava, Peter Lang, Frankfurt am Main, 2013.

[PAS2] Remarks on one type of uniform distribution, Unif. Distrib. Theory 2 (2007), $79-92$.

[Ra] RAUZY, G.: Propriétés statistique de suites arithmétiques. Collection SUP, Le Mathématicien 15, Presses Universitaires de France, Paris, 1976.

[SP] STRAUCH, O.-PORUBSKÝ, Š.: Distribution of Sequences. A Sampler. Slovak Academy of Sciences Bratislava, Peter Lang, Frankfurt am Main, 2005.

[ST] A distribution of sequences, a theory, draft version, Mathematical Institute, Slovak Academy of Sciences, Bratislava, 2018.

[WEY] WEYL, H.: Über die Gleichverteilung von Zahlen mod. Eins, Math. Ann. 77 (1916), 313-352. (In German)

Received April 30, 2018

Department of Mathematics
Faculty of Education
University of Trnava
Priemyselná 4
SK-917-01 Trnava
SLOVAKIA
E-mail: milan.pasteka@truni.sk 